IZA DP No. 8709

Sleepiness, Choice Consistency, and Risk Preferences

Marco Castillo

David L. Dickinson

Ragan Petrie

December 2014

Forschungsinstitut zur Zukunft der Arbeit Institute for the Study of Labor 


\title{
Sleepiness, Choice Consistency, and Risk Preferences
}

\author{
Marco Castillo \\ ICES, George Mason University \\ David L. Dickinson \\ CERPA, Appalachian State University \\ and IZA \\ Ragan Petrie \\ ICES, George Mason University
}

Discussion Paper No. 8709

December 2014

\author{
IZA \\ P.O. Box 7240 \\ 53072 Bonn \\ Germany \\ Phone: +49-228-3894-0 \\ Fax: +49-228-3894-180 \\ E-mail: iza@iza.org
}

Any opinions expressed here are those of the author(s) and not those of IZA. Research published in this series may include views on policy, but the institute itself takes no institutional policy positions. The IZA research network is committed to the IZA Guiding Principles of Research Integrity.

The Institute for the Study of Labor (IZA) in Bonn is a local and virtual international research center and a place of communication between science, politics and business. IZA is an independent nonprofit organization supported by Deutsche Post Foundation. The center is associated with the University of Bonn and offers a stimulating research environment through its international network, workshops and conferences, data service, project support, research visits and doctoral program. IZA engages in (i) original and internationally competitive research in all fields of labor economics, (ii) development of policy concepts, and (iii) dissemination of research results and concepts to the interested public.

IZA Discussion Papers often represent preliminary work and are circulated to encourage discussion. Citation of such a paper should account for its provisional character. A revised version may be available directly from the author. 


\section{ABSTRACT \\ Sleepiness, Choice Consistency, and Risk Preferences}

We investigate the consistency and stability of individual risk preferences by manipulating cognitive resources. Participants are randomly assigned to an experiment session at a preferred time of day relative to their diurnal preference (circadian matched) or at a nonpreferred time (circadian mismatched) and choose allocations between two risky assets (using the Choi et al., 2007, design). Consistency of behavior of circadian matched and mismatched subjects is statistically the same, however mismatched subjects tend to take more risks. We conclude that, consistent with several theories, preferences are rational yet can change depending on state-level cognitive resources.

\section{NON-TECHNICAL SUMMARY}

It is generally known that sleepiness affects one's choices when facing risk. It is not known, however, whether behavioral changes due to sleepiness imply a change in the frequency of irrational or inconsistent choices, or whether the changes are due to a basic change in preference towards risk. We manipulate sleepiness by altering the time of day when we administer a monetary risk task to validated morning-type and evening-type individuals. We find that decisions at off-peak times in one's circadian cycle show an increased preference for risk, but the consistency of choices (i.e., rationality) does not differ. This result has important implications. Those who must make decisions while sleepy, such as shift workers, may be susceptible to this tendency to favor risk.

JEL Classification: C91, D81

Keywords: $\quad$ sleep, risky preference, choice consistency

Corresponding author:

David L. Dickinson

Department of Economics

Appalachian State University

Boone, NC 28608

USA

E-mail: dickinsondl@appstate.edu 


\section{Introduction}

There is growing evidence that individual risk attitudes, as measured by economic experiments, vary across people and circumstances. These include life-cycle changes, traumatic personal or family experiences (Voors et al, 2012; Malmendier and Nagel, 2011; Callen et al, 2012), physical conditions (Garbarino et al, 2011; Wozniak et al, 2010), priming and framing (Benjamin et al., 2010), cognitive ability (Dohmen et al., 2010; Burks et al, 2009; Benjamin et al, 2012), the different way in which some may bracket choices (Read et al, 1999) and by ones' genetic makeup (Cesarini et al, 2009).

In this paper, we examine if temporary challenges to cognitive functioning yield choices that are consistent with rationality or whether differences in decisions are due to lapses in rational behavior broadly defined. In particular, we investigate whether a sleepiness manipulation through circadian mismatch, which is shown to be associated with impairment of cognitive abilities (Bodenhausen, 1990; Kruglanski and Pierro, 2008; Dickinson and McElroy, 2012), produces changes in preferences while maintaining consistency of behavior. A common assumption in standard economic models (e.g. Arrow-Debreu state-dependent preferences model, Mas-Collel et al, 1995), as well as behavioral models (Tversky and Kahnemen, 1992; Koszegi and Rabin, 2007; Becker and Murphy, 1988), is that changes in preferences can occur without the loss of rationality. Our experimental design provides empirical evidence to investigate this assumption.

Circadian timing of decisions is a natural environment to test the stability and consistency of preferences. First, sleepiness has been widely studied in the sciences, and its effects on performance in many domains are well documented and understood. ${ }^{1}$ Second, it is a physical condition commonly experienced by most people at some, or many, period(s) of their lives. Because of this, circadian mismatch, compared to other ways to temporarily deplete cognitive resources, is a manipulation that is less likely to generate inconsistencies in behavior due to learning or adaptation to the circadian mismatch. This is important because such learning would confound an examination of preference consistency across states. Third, results

\footnotetext{
${ }^{1}$ Total sleep deprivation studies are a more common approach to studying how sleepiness affects performance and decisions. However, circadian mismatch is a milder, and arguably more externally valid, way to study sleepiness of the sort commonly experienced by real-world decision makers.
} 
from this type of environment should be relevant to policy. Understanding whether risky choice decisions while sleepy are rational or not and if preferences change could help inform the design of institutions and policies.

Our research protocol is designed to minimize issues of selection and allow interpretation of our results as due to temporary cognitive resource depletion. We start by collecting survey information, which includes a validated measure of one's diurnal preference, on a large number of participants. The data are then used to identify two classic diurnal preference groups: those who are naturally most alert in the morning and those who are naturally most alert in the evening. These morning types and evening types were then randomly assigned to one of two session times: early morning or late night. This produced two treatments, participants who were "matched" in terms of their circadian rhythm (e.g. morning type in a morning session and evening type in an evening session) and circadian "mismatched" (e.g. morning type in an evening session and evening type in a morning session). Participants assigned to one session time were not allowed to switch to the other. Compliance with session assignment was voluntary, and importantly, we find no significant differences in compliance across treatment conditions. Participants were allowed to take all the time they needed to make their decisions, and this was done to allow participants the opportunity to express their preferences unconstrained by time.

Our results show a significant treatment effect on risk decisions, and as mentioned above, this is not due to selection or compliance across treatments. Circadian mismatched participants have higher certainty equivalents for different risky asset bundles, indicating they are less risk-averse. Also, the variance in risky asset investment is larger for these subjects, showing a tolerance for more variability in payments.

While the manipulation clearly worked as designed and affected preferences, it did not alter the likelihood a subject behaved rationally. Adherence to the generalized axiom of revealed preference (GARP) is identical between mismatched and matched participants. ${ }^{2}$ As a

\footnotetext{
${ }^{2}$ Similarly, deviations from expected utility theory (EU) or more general models of non-expected utility (NEU) behavior are also statistically similar across groups. We also examine whether subjects violate payoff dominance in making choices and, while both groups display some violations, there is no difference in violations between groups. In our data, consistency with rationality is robust.
} 
result, the estimated behavioral differences result cannot be attributed to an increase in noisiness of the data following circadian mismatch because an increase in noise would manifest in increased violations of choice consistency of one sort or another. All of this suggests that preferences can be altered without altering adherence to rational behavior however defined.

Our paper contributes to the literature by showing that a slight manipulation of physical conditions, to produce a temporary challenge to cognition, produces changes in risk attitudes without producing a breakdown of rationality. Our results contrast with those of Dohmen et al. (2010), Burks et al (2009), and Frederick (2005), who show that higher levels of permanent cognitive ability are correlated with increased propensity to take risk. Our manipulation leads to higher certainty equivalents for mismatched subjects, indicating an increased preference for monetary risk when sleepy. Our experiment was not designed to identify the mechanism causing these effects, however, our results do show that the relationship between alertness and preferences is causal.

To our knowledge, this is the first paper to show that changes in preferences can occur without loss of rationality. The closest paper to ours is that of Burghart et al., (2012), who examine behavioral effects of alcohol intake but conclude that it does not impair rational decision-making. In our data, we do identify a change in risk preference in spite of no difference in rationality. ${ }^{3}$

In the next section, we describe the experimental design and the cognitive resource manipulation. We then turn to results, first by confirming that our manipulation worked, then examining rationality and choice behavior in the risk task. Finally, we conclude.

\section{Experimental Design}

\subsection{The Risky Choice Experiment Environment}

\footnotetext{
${ }^{3}$ Our design includes 50 decisions per subject and provides more statistical power, which increases our confidence in the finding that rationality is not altered by our manipulation. Also, in our design, participants were randomly assigned to treatments (circadian mismatched or matched), and this reduces the possibility that participants selfselect into the experiment based on certain characteristics (e.g. rationality).
} 
We follow the design of Choi et al (2007) for the risky choice task, which generates a rich set of individual-level data. ${ }^{4}$ In each decision round, subjects are asked to allocate tokens between two different accounts: $X$ and $Y$. Tokens in account $X$ only generate a payoff for the subject if account $X$ is randomly chosen by the computer at the end of that decision round. Similarly, tokens in account $Y$ only pay if account $Y$ is randomly selected. We implement the "symmetric" treatment design of Choi et al (2007) with a common knowledge 50\% probability that either account $X$ or $Y$ will be chosen. Figure 1 shows a sample stimulus where the subject makes an allocation choice on a computer interface by using a mouse-driven pointer to drag point $C$ along the line $A B$ to their desired choice location (including the endpoint locations, if desired). An allocation such as point A or point $B$ is a risky choice with all tokens placed in one account. Thus, the subject would only receive a payoff if the computer randomly selects the account where all the tokens are allocated. An intermediate allocation of tokens, such as point C in Figure 1, places some tokens in each account, which guarantees the subject a smaller, but sure, payoff in both states of the world. A choice along the $X=Y$ line in Figure 1 is a perfectly safe portfolio that guarantees the same payoff no matter which state of the world applies.

The experiment consists of 50 decision rounds (i.e., 50 different stimuli) where the slope and intercept of the $A B$ line are randomly determined for each stimulus. ${ }^{5}$ After all 50 rounds, one round is randomly selected for payment, and each round has an equal probability of being chosen. The randomly selected payoff-round, the computer's random selection of account $\mathrm{X}$ or $\mathrm{Y}$, and the subject's allocation decision for that round determines the subject's payoffs.

\subsection{The Cognitive Resource Manipulation}

We use a circadian match/mismatch protocol to represent a temporary challenge to cognitive resources. While there may be other ways to temporarily deplete resources, our

\footnotetext{
${ }^{4}$ We are grateful to Sachar Kariv for providing us with the code for the experiment task.

${ }^{5}$ For the first eight sessions, the $X$ and $Y$ intercepts were constrained to lie in the $[50,100]$ interval. For the latter eight sessions, in order to generate more extreme relative prices, the budgets were chosen among the set of lines that intersect at least one axis at or above the 50-token level, but below 100, and intersect both axes at or below the 100-token level. The initial starting point for the mouse-pointer along each budget line was also randomly determined. See Choi et al., (2007) for full details.
} 
method has broad applicability to circumstances encountered in daily life, has been previously used and validated in the literature, and is relatively easy to administer.

Previous research has shown that single-vehicle accidents increase at times of the day where the typical circadian rhythm dictates sleepiness due to natural release of melatonin (Coren, 1996). In controlled experiment settings, researchers have found that sleep deprived individuals take on more risk than well-rested individuals when choosing between risky lotteries (McKenna et al., 2007). In a different risky choice task, Venkatraman et al, (2007) found neural effects in sleep-deprived subjects even in the absence of behavioral effects. Though sleep loss and circadian timing may both contribute to depleted cognitive resources with symptomatic sleepiness, a 24-hour total sleep deprivation protocol likely depletes cognitive resources to a greater extent than what individuals commonly experience on a daily basis and may therefore not be applicable to a large segment of the population. Decision-making at sub-optimal times of the day is more externally valid, and hence motivates our choice of the circadian mismatch protocol.

Explicit circadian mismatch protocols like we propose have been used in behavioral research to some extent, but this area is relatively unexplored. Bodenhausen (1990) showed that individuals are more likely to use stereotypes in making judgments when at circadian mismatched times, and Kruglanski and Pierro (2008) reported an increased use of the psychological transference effect among subjects tested at circadian mismatched times. Dickinson and McElroy (2010) and Dickinson and McElroy (2012) used two distinct protocols to manipulate the circadian timing of decision in guessing games, and find that choices made at circadian mismatched times generally produce outcomes farther from the predicted Nash equilibrium. Though limited, the extant literature on circadian mismatch effects is consistent with the hypothesis that circadian mismatch alters decision-making in a way consistent with cognitive resource depletion. ${ }^{6}$

\footnotetext{
${ }^{6}$ To be more specific, depletion of cognitive resources would disproportionately affect executive function. The behavioral effects reported in the aforementioned studies either involve increased reliance on heuristics (i.e., stereotyping and transference effect) or decreased ability to engage in strategic reasoning (i.e., the guessing game), both of which are consistent with reduced engagement of deliberate thought regions of the brain that rely on fully intact cognition.
} 
To implement the circadian mismatch protocol for our current study, we first administer a large-scale online survey at two academic institutions. The objective of the survey is to generate a database of individuals for whom we have a validated measure of their diurnal preference, which is assessed in the survey using the short form of the morningnesseveningness questionnaire, henceforth rMEQ (Adan and Almiral, 1991). The rMEQ classifies individuals on a scale of 4-25, with morning-types having rMEQ score from 18-25 and eveningtypes having rMEQ score from 4-11. While this diurnal preference measure is based on selfreports of the subjects, it has been validated against physiological data on oral temperatures (see Horne and Östberg, 1976) and is a standard tool in circadian research.

From our database, we recruit morning-types and evening-types, who we had randomly assigned, ex ante, to participate in either a morning (7:30 a.m.) or an evening (10:00 p.m.) experiment session. This resulted in $57 \%$ of our sample being circadian matched for the risky choice experiment. ${ }^{7}$ If a subject could not participate in the randomly assigned time-slot (morning or evening), the subject was not allowed the option of the alternative time-slot-an alternative time-slot was not even mentioned in recruitment. This aspect of our design eliminates selection into treatments and allows for a more causal interpretation of cognitive resource depletion on outcomes. Importantly, we find no evidence of selection in show-up rates across the matched and mismatched subjects. The proportion of subjects who actually showed up for the session they signed up for is not significantly different across our matched and mismatched subjects (the p-value of a Chi-square test of difference in distribution is $0.516) .^{8}$

We recruited a total of 202 subjects for this study. Table 1 shows the distribution of our sample across experiment locations for each design cell. The experiment sessions lasted just over an hour, and included the risky choice task administration as well as a few short survey

\footnotetext{
${ }^{7}$ Due to the rarity of true morning-type subjects-less than $10 \%$ in young adult populations are morning-types (see Chelminski et al, 2000) - we extend our rMEQ cutoff to include rMEQ scores of 15-17. To compensate, we only recruit the more extreme (and still abundant) evening-type subjects with rMEQ scores from 4-10. In this way, our sample is still drawn from the tails of the rMEQ distribution and eliminates the same amount of support from the non-tail portion of the $\mathrm{rMEQ}$ distribution compared to if we had used the traditional morning-type cutoff ( $\mathrm{rMEQ}=18$ ) but included non-extreme evening types ( $\mathrm{MMEQ}=11$ ) in our sample.

${ }^{8}$ One hundred and thirty-seven matched subjects signed up for experimental sessions, and 114 showed up. One hundred and ten mismatched subjects signed up for experimental sessions, and 88 showed up.
} 
instruments to elicit self-reported measures of recent sleep habits. Average subject payoffs were $\$ 22.56$ (s.d. \$9.61), which includes a \$5 show up fee.

\section{Results}

\subsection{Summary Statistics and Manipulation Check}

Table 2 shows relevant summary statistics of our sample. Pre-experiment survey data refers to responses from the online sleep survey administered as a way of building our database of morning-type and evening-type subjects. Pre-experiment survey responses would have been given several days to several weeks before the decisions experiments. Table 2 includes summary statistics for the same questions asked in the pre-experiment survey and after subjects had completed the risky choice task but before payments were revealed. These include self-reports of the subjects' average nightly sleep over the 7-days prior to the response, average sleep the night prior to the response, one's subjective optimal hours of nightly sleep required for peak performance, and the Epworth Sleepiness scale used commonly in sleep research (a measure of trait-level sleepiness, or chronic fatigue). As can be seen in the top panel, there are no significant differences in any of these descriptive measures between the circadian matched and mismatched subjects in the pre-experiment survey. In the postexperiment survey, matched subjects report having more sleep over the previous week and the night prior to the experiment.

Because our objective is to introduce a randomized assignment of cognitive resource availability, we present evidence that our circadian manipulation was successful. The manipulation checks in Table 2 reveal that circadian mismatched subjects, who presumably have depleted cognitive resources, report significantly higher state-level sleepiness and have higher subjective sleep deprivation (optimal amount of sleep less sleep previous night). These are all consistent with what we expect from a random assignment of a "sleepiness" manipulation. ${ }^{9}$

\footnotetext{
${ }^{9}$ There is no a priori reason why sleepy subjects would be expected to make faster or slower decisions. Nonetheless, mismatched and matched subjects are no different in the length of time it takes to make decisions during the experiment.
} 
We also confirm that, on average, circadian matched and mismatched subjects faced similar menus-average intercepts of randomly generated budget lines in Fig. 1 do not significantly differ in mean or variance across. ${ }^{10}$

In sum, we validate that our sleepiness manipulation worked and the decision environment for our matched and mismatched subjects was the same.

\subsection{Consistency of Behavior}

We look first at rationality and then choices in the risk task. We test for consistency of choices with rationality for matched and mismatched subjects using several measures of rational behavior. Specifically, we test if subjects satisfy the Generalized Axiom of Revealed Preferences (GARP: Afriat 1972; Varian, 1983) and payoff dominance. ${ }^{11}$ This examination of choice rationality provides a test of whether there is an increase in the noise of the choice data when sleepy. Noisier choice data would result in increased violations of GARP and payoff dominance. As we will see below, this is not the case.

Figure 1 presents the distribution of the Critical Cost to Efficiency Index, CCEI, (Afriat, 1972) for matched and mismatched subjects. This index measures how much budget constraints would need to be adjusted to eliminate all violations of GARP. As a point of reference as to how the CCEI measures rational choices, the figure also shows the distribution of CCEI for random choices. This distribution is shown in light bars, and the distribution for subject choices is shown in blue bars. The two distributions are significantly different (Chisquare test of difference in distribution $p$-value $=0.000$ ) and show that subject choices in the experiment are not random. ${ }^{12}$

Looking at the CCEI's of subjects, Figure 1 shows that 13 percent of matched and mismatched subjects satisfied GARP without having to modify any budget $(C C E I=1)$. An

\footnotetext{
${ }^{10}$ A Kolmogorov-Smirnov test for difference in the distribution of the maximum intercept (max amount a person can put in one asset) that matched and mismatched subjects saw shows no significant difference. The test was run separately for the first 8 sessions and the last 8 sessions because these differed in the budget generation process (see footnote 4). The $p$-value for the first 8 sessions is 0,674 and for the last 8 sessions is 0.868 .

${ }^{11}$ Additional tests of rationality (expected utility, disappointment aversion or cumulative prospect theory) produce the same results. Tests and results are available from the authors upon request.

${ }^{12}$ We only consider positive prices in all the analyses in the paper. Out of the 10,100 choices made in the experiment (50 choices per subject x 202 subjects), 10,094 were with strictly positive prices.
} 
additional 21 percent of the matched subjects and 22 percent of the mismatched subjects require a small change in the budgets to satisfy GARP (CCEI above 0.999). All told, 78 percent of the matched subjects and 83 percent of the mismatch subjects have indices strictly above 0.9 . Indeed, the distribution of the CCEI is not significantly different between these two groups (rank sum test of difference in distributions $p$-value $=0.7805$ ). That is, slight cognitive resource depletion does not cause an increase in the distant to rationality, as measured by the CCEI.

Adherence to payoff dominance makes clear predictions in the context of this choice task. Namely, if one considers Fig. 1, we can see that for any set of relative asset prices other than 1, a subject should never choose along the segment of the budget line on the short side of the safe bundle line. In other words, any choice off the $X=Y$ line represents increased risk, but moving away from $\mathrm{X}=\mathrm{Y}$ onto the longer segment of the budget line increases expected payoff compared to choosing the short side of the budget line, which increases risk but decreases expected payoff. Thus, all choices on the short side (e.g., above the $X=Y$ line in Fig. 1) of the budget line violate payoff dominance. Our data set provides ample observations to examine violations of payoff dominance in our two experimental groups.

Table 3 presents the contingency table of violations of payoff dominance for the steep versus flat budget constraints (the rows), and it does so for the case of the entire data set as well as subsets of the data for which relative prices are quite close (the columns). The importance of investigating relatively close price ratios is because violations of dominance are less severe in those cases, at least in terms of the magnitude of the expected payoff loss. Observing significant differences across matched and mismatched participants for close price ratios would be a strong test for noisy decision making. Fisher's exact tests are performed for each column represented in Table 3.

Two things are clear from Table 3. First, there are a significant number of violations of payoff dominance. It is violated roughly $1 / 3$ of the time for the set of all budget constraints and, not surprisingly, dominance is violated more frequently as we constrain the data to the set of relative prices closer to one (i.e., the $\left|\ln \left(\frac{\boldsymbol{P}_{\boldsymbol{x}}}{\boldsymbol{P}_{Y}}\right)\right|<\mathbf{0 . 1 0}$ and $\left|\boldsymbol{\operatorname { l n }}\left(\frac{\boldsymbol{P}_{\boldsymbol{x}}}{\boldsymbol{P}_{Y}}\right)\right|<\mathbf{0 . 0 5}$ subsamples). That is, as expected, violations increase as the cost of a violation decreases. The second observation from Table 4 is that there is no significant difference in propensity to violate dominance 
between circadian matched and mismatched participants. Violations of dominance are actually uniformly lower among the circadian mismatched group, but the Fisher's exact tests show no statistically significant differences. These results complement the rather extensive examination of consistency with other definitions of rationality, but all tests in this section point to the same result.

RESULT 1: Cognitive resource depletion via circadian mismatch does not affect choice consistency

In summary, we find that distance to rational behavior across circadian matched and mismatched subjects is similar regardless of the test of rational behavior we conduct. When testing the data's consistency with GARP, for which there are some CCEI benchmarks in the literature, both our circadian matched and mismatched groups would be deemed "rational." We now examine if consistency in behavior, whether cognitively challenged or not, also implies that choices in the risk task are the same.

\subsection{Choices in the Risk Task}

In light of the absence of a discernible difference in consistency of behavior, in this section, we investigate whether the circadian mismatch manipulation affects risky choices. We look at the distribution of asset investments, from which we calculate certainty equivalents for

subjects. These certainty equivalents constitute a theoretically valid measure of risk preference.

Because deviations from expected utility theory might manifest through nonlinear responses to prices we take these factors into consideration in the analysis that follows. In particular, subjects might choose a distribution of assets that favors constant payoffs. Therefore, small variations in relative prices will have a different impact on asset allocations than large changes in relative prices. It will be important to examine behavior in these extremes.

Figure 3 shows the distribution of the proportion of the budget share in asset $Y$ for all relative prices for matched and mismatched subjects. Matched subjects tend to more frequently choose asset allocations that secure equal payoffs across states of nature. Thus, 
matched subjects choose the safe bundle more frequently, which is an indication of increased risk aversion relative to circadian mismatched subjects. ${ }^{13}$ The significance of this result is tested and shown in Table 4. This table shows the results of an interquantile regression of budget share on a dummy variable for being a mismatched subject. The interquantile range of budget share in the $\mathrm{Y}$ asset is 8.5 percentage points larger than that of matched subjects. Mismatched subjects are significantly more likely to make riskier investments.

To more rigorously assess differences in risk aversion, we calculate non-parametric certainty equivalents for the matched and mismatched subject groups. The certainty equivalent for a particular risky lottery is estimated as the highest payoff "safe bundle" (i.e., equal payoffs across states) that would be less preferred to the non-equal payoff "risky" lottery and still be consistent with GARP. That is, we look for all sets of relative prices that would support the "risky" lottery without violating GARP and pick the set of relative prices that include the highest possible "safe bundle." This highest "safe bundle" is our certainty equivalent measure. So, the more risk averse an individual is the lower the "safe bundle" would be to make them switch. This means that more risk averse individuals would have lower certainty equivalents. Finally, calculating certainty equivalents requires subjects to be consistent. So, since many subjects have some violation of GARP, we adjust the revealed preferred to relation according to the subject's CCEI. In particular, all the calculations define a bundle $x$ at prices $p$ to be revealed preferred to $y$ if $\operatorname{CCEI}(i)^{*} p^{*} x \geq p^{*} y$, where CCEI(i) is subject i's CCEI. ${ }^{14}$

The results from calculating these certainty equivalents are shown in Figure 4. The average certainty equivalent (CE) for matched and mismatched subjects are calculated for each asset bundle, and Figure 4 shows the difference of these CEs between matched and mismatched subjects relative to the expected value of the lottery. This shows how much larger the CE is for mismatched compared to matched subjects in percentage points. The general path

\footnotetext{
${ }^{13}$ Given the mouse-driven graphical choice interface, one might think that sleepy subjects would be less likely to choose safe asset bundles due to motor skill deficits resulting from fatigue. We note that this is not likely the case in our data, however, because that argument would imply these same sleepy subjects are more likely to choose extreme border asset bundles. This is not the case in our data.

${ }^{14}$ As a robustness check, we evaluate our results on the differences in certainty equivalents between matched and mismatched subjects by also constraining the sample to subjects whose CCEI is close to one. Our main result, that matched subjects are more risk averse, still holds. The result is no longer statistically significant though, and that is a reflection of the smaller number of observations in the constrained sample.
} 
of where these differences lie is along the range of actual available lotteries seen in the experiment. What is clear from these data is that mismatched individuals have higher calculated certainty equivalents than matched individuals, especially for extreme lotteries that are far from the sure payoff lottery. In other words, matched subjects are more risk averse than mismatched subjects.

We test for the significance of these differences in Table 5. This table shows results for Tobit regressions of the CE on a dummy variable for being mismatched for various sets of relative prices. ${ }^{15}$ The first column includes all the data and shows that mismatched subjects have a 4.4 percentage point larger CE than matched subjects. The second column confirms this results when the data are restricted to include relative prices strictly different from one. Columns 3-6 further restrict the data to include only progressively more extreme relative prices. Mismatched subjects have higher CE than matched subjects, but this is only significant in a onesided test. This leads to our second result:

RESULT 2: Cognitive resource depletion via circadian mismatch leads to higher certainty equivalents (i.e., increased preference for risk).

As a further check, we examine how price responsiveness varies across groups. Table 6 shows regressions of price elasticity on relative prices, a dummy for being mismatched and an interaction term of relative prices on being mismatched. The significant negative coefficient on the interaction term indicates that mismatched subjects appear more sensitive to changes in relative asset prices than matched subjects. This implies that more steep (flat) budget lines in Fig 1 lead mismatched subjects to alter their chosen asset bundles even further towards the cheaper asset than would a matched subject. Another interpretation of this result is that mismatched subjects are less sensitive to the risk of the low (zero) payoff than matched subjects when budget lines reflect large relative price differences. It is also the case that any budget slope other than -1 would lead a risk neutral expected value maximizing subject to

\footnotetext{
${ }^{15}$ The Tobit regression takes the calculated certainty equivalent for an individual for a given lottery and regresses it on a dummy variable for being mismatched. There are 361 possible generated lotteries for each individual and 202 individuals, yielding 72,922 observations, and the regressions cluster at the individual level.
} 
choose a corner solution. The Table 6 result is therefore consistent with the hypothesis that mismatched subjects take more risks because they are more desensitized to risk, which is a result reported previously in the literature using totally sleep deprived subjects (McKenna et al., 2007).

While mismatched subjects are more risk taking, does this result in payoff differences across the two groups? In our experiments, subjects are paid based on one randomly chosen trial, so we examine expected payoff differences given a subject's 50 trails of risky choices. Doing so, we find that mean expected payoffs are higher for circadian mismatched subjects, however, the results are not statistically significant. ${ }^{16}$ We find no evidence that the increased tendency of circadian mismatched subjects to take risk benefits or harms payoff outcomes.

In sum, while the emerging literature has found that individuals with lower levels of permanent cognitive abilities are more risk averse, we find that our sleepiness manipulation leads to lower risk aversion as measured by certainty equivalent. This result is consistent with other literature examining extreme forms of temporary cognitive resource depletion effects on incentivized risky choice tasks, such as total sleep deprivation (e.g., McKenna et al., 2007) or intoxication (e.g., Lane et al., 2004). Importantly, despite the shift in risk attitudes, we do not find any significant difference in decision-making rationality resulting from circadian mismatch, under several alternative definitions of consistency.

\section{Conclusions}

In this paper we investigate how a particular form of cognitive resource depletion impacts choice consistency and outcomes in a risky choice task. The task (Choi et al., 2007) generates data which allow us to evaluate choice consistency with respect to several different measures of rationality. As a result, our contribution is that we are able to establish whether differences in preferences over risky asset bundles are the result of "irrationality", or whether they are the result of state-dependent preferences. We find that they are due to the latter. The circadian mismatch protocol we implement to manipulate cognition is not only effective but

\footnotetext{
${ }^{16}$ We test this by running quantile regressions of expected payoff on a dummy variable for being mismatched. While expected payoffs are higher for mismatched subjects, there is no significant difference for the $25 \%, 50 \%$ and 75\% quantile. Errors are bootstrapped 1000 times.
} 
externally valid and similar to what decision makers face in field environments. While much of the recent literature has focused on how permanent cognitive levels may correlate with risk preferences, we address how temporary fluctuations in available cognitive resources may affect choice, independent of permanent abilities.

Our results are significant and reveal evidence that randomly assigned circadian mismatch subjects are no less rational than matched subjects, and yet preferences for risk shift. Specifically, we have shown that choices are no more or less consistent with GARP or payoff dominance theories as a result of the subject being circadian mismatched. And yet, these mismatched subjects are more willing to accept risky asset bundles compared to matched subjects.

This is an important result with practical and policy implications, especially if one considers that many real-world decision makers face even more serious bouts of sleepiness than the relatively mild manipulation we implement. In the realm of monetary risk choice, sleep deprivation is estimated to affect over $25 \%$ of workers in the financial and insurance industries (CDC, 2012). In such industries, any increased tendency to take risk may have significant consequences. In other occupations, risky choice may not involve explicit monetary risk (e.g., air traffic controllers, long-haul trucking, medical practice, or emergency service workers), but sleepiness is commonplace and of great concern to policymakers establishing regulations that may involve prescribed rest or time-off to avoid sleep deprivation or limit shift work.

If one considers the other various forms of temporary cognitive challenges we often face (e.g., multi-tasking, stress, time pressure), this research may have even more wide reaching implications. We leave it to future research to establish the relationship, if any, between various distinct forms of cognitive resource manipulations, or between cognition effects on monetary risk preference versus other choice domains. Nonetheless, it is clear that this area of research is fertile ground for studying choice in the real world where cognitive resources are not uniformly available at all times. 
Acknowledgements: The authors thank David Bruner, Olivier l'Haridon, participants at the Economic Science Association meetings, and seminar participants at the University of Rennes and Appalachian State University for helpful comments on earlier drafts of this paper.

\section{REFERENCES}

Adan, A., and H. Almiral. 1991. "Horne and Ostberg morningness-eveningness questionnaire: A reduced scale." Personality and Individual Differences, 12: 241-253.

Afriat, Sidney N. 1972. "Efficiency estimation of production function." International Economic Review, 8(1): 67-77.

Banks, James, Cormac O’Dea, and Zoë Oldfield. 2010. “Cognitive function, numeracy and retirement saving trajectories." The Economic Journal, 120(548): F381-F410.

Becker, Gary and Kevin Murphy. 1988. "A Theory of Rational Addition." Journal of Political Economy. 96(4), 675-700.

Benjamin, Daniel, Sebastian Brown and Jesse Shapiro. 2012. "Who is 'Behavioral'? Cognitive Ability and Anomalous Preferences," Journal of the European Economic Association. Forthcoming.

Benjamin, Daniel, James Choi and Joshua Strickland. 2010. "Social Identity and Preferences." American Economic Review. 100(4), 1913-1928.

Bodenhausen, Galen V. 1990. "Stereotypes as judgmental heuristics: Evidence of circadian variations in discrimination." Psychological Science, 1: 319-322.

Burghart, Daniel R., Paul W. Glimcher, and Stephanie C. Lazzaro. 2012. "An expected utility maximize walks into a bar..." Working paper, New York University.

Burks, Stephen, V., Jeffrey P. Carpenter, Lorenz Goette, and Aldo Rustichini. 2009. "Cognitive skills affect economic preferences, strategic behavior and job attachment." Proceeding of the National Academy of Sciences, 106(19): 7745-7750.

Callen, Michael, Mohammad Isaqzadeh, James Long and Charles Sprenger. 2012. "Violence and Risk Preferences: Artefactual and Experimental Evidence from Afghanistan," Working Paper. Stanford University.

Centers for Disease Control and Prevention. 2012. Morbidity and Mortality Weekly Report. April 27, 61(16): 281-285. Accessed at http://www.cdc.gov/mmwr/preview/mmwrhtml/mm6116a2.htm September 27, 2012. 
Cesarini, David, Christopher Dawes, Magnus Johannesson, Paul Lichtenstein and Bjorn Wallace. 2009. "Genetic Variation in Preferences for Giving and Risk Taking," Quarterly Journal of Economics. 124(2), 809-842.

Chelminski, Iwona, Thomas V. Petros, Joseph J. Plaud, and R. Richard Ferraro. 2000. "Psychometric properties of the reduced Horne and Ostberg questionnaire." Personality and Individual Differences, 29(3): 469-478.

Choi, Syngjoo, Raymond Fisman, Douglas Gale, and Shachar Kariv. 2007. "Consistency and heterogeneity of individual behavior under uncertainty." American Economic Review, 27(5): 1921-1938.

Coren, Stanley. 1996 "Daylight savings time and traffic accidents." New England Journal of Medicine, 334: 924.

Dickinson, David L., and Todd McElroy (2012) "Circadian effects on strategic reasoning." Experimental Economics. 15(3): 444-459. DOI 10.1007/s10683-011-9307-3

Dickinson, David L, and Todd McElroy. 2010. "Rationality around the clock: Sleep and time-ofday effects on guessing game responses." Economic Letters, 108: 245-248.

Dohmen, Thomas, Armin Falk, David Huffman, and Uwe Sunde. 2010. “Are risk aversion and impatience related to cognitive ability?" American Economic Review, 100(June): 12381260.

Fleissig, Adrian R., and Gerald A. Whitney. 2005. "Testing for the significance of violations of Afriat's inequalities." Journal of Business \& Economic Statistics, 23(3): 355-362

Frederick, Shane. 2005. "Cognitive reflection and decision making." Journal of Economic Perspectives, 19: 25-42.

Garbarino, Ellen, Robert Slonim, Justin Sydnor. 2011. “Digit Ratios (2D:4D) as Predictors of Risk Decision Making for Both Sexes," Journal of Risk and Uncertainty. 42(1), 1-26.

Gul, Faruk. 1991. “A Theory of disappointment aversion.” Econometrica, 59(3): 667-86.

Horne, Jim A, and Olov Östberg. 1976. A self-assessment questionnaire to determine morningness-eveningness in human circadian rhythms. International Journal of Chronobiology, 4: 97-110. 
Koszegi, Botond and Matthew Rabin. 2007. "Reference-Dependent Risk Attitudes." American Economic Review. 97(4), 1047-1073.

Kruglanski, Arie W., and Antonio Pierro. 2008. "Night and day, you are the one. On circadian mismatches and the transference effect in social perception." Psychological Science, 19(3): 296-301.

Lane, Scott D., Don R. Cherek, Cynthia J. Pietras, and Oleg V. Tcheremissine. 2004. "Alcohol effects on human risk taking." Psychopharmacology, 172: 68-77.

Malmendier, Ulrike and Stefan Nagel. 2011. "Depression Babies: Do Macroeconomic Experiences Affect Risk Taking?" Quarterly Journal of Economics. 126(1), 373-416.

Mas-Collel, Andreu, Michael Whinston and Jerry Green. 1995. Microeconomic Theory. Oxford University Press, New York.

McElroy, Todd., and David L. Dickinson. 2010. "Thoughtful days and valenced nights: How much will you think about the problem?" Judgment and Decision Making, 5(7): 516-23.

McKenna, Benjamin S., David L. Dickinson, Henry J. Orff, and Sean P. A. Drummond. 2007. "The effects of one night of sleep deprivation on known-risk and ambiguous-risk decisions." Journal of Sleep Research, 16: 245-252.

Read, Daniel. George Loewenstein, and Matthew Rabin. 1999. "Choice bracketing." Journal of Risk and Uncertainty, 19(1-3): 171-97.

Tversky, Amos, and Daniel Kahneman. 1992. "Advances in prospect theory: Cumulative representation of uncertainty." Journal of Risk and Uncertainty, 5(4): 297-323.

Varian, Hal R. 1983. "Nonparametric tests of models of investor behavior." Journal of Fainancial and Quantative Analysis, 18(3): 269-278.

Venkatraman, V., Y.M.L. Chuah, and S.A. Huettel. 2007. "Sleep deprivation elevates expectation of gains and attenuates response to losses following risky decisions." Sleep, 30: 603-609.

Voors, Maarten, Eleonora Nillesen, Philip Verwimp, Erwin Bulte and Robert Lensink. 2012. "Violent Conflict and Behavior: A Field Experiment in Burundi." American Economic Review. 102(2), 941-964.

Wozniak, David, William Harbaugh and Ulrich Mayr. 2010. "The Menstrual Cycle and Performance Feedback Alter Gender Differences in Competitive Choices," Working Paper. University of Oregon. 


\section{FIGURES AND TABLES}

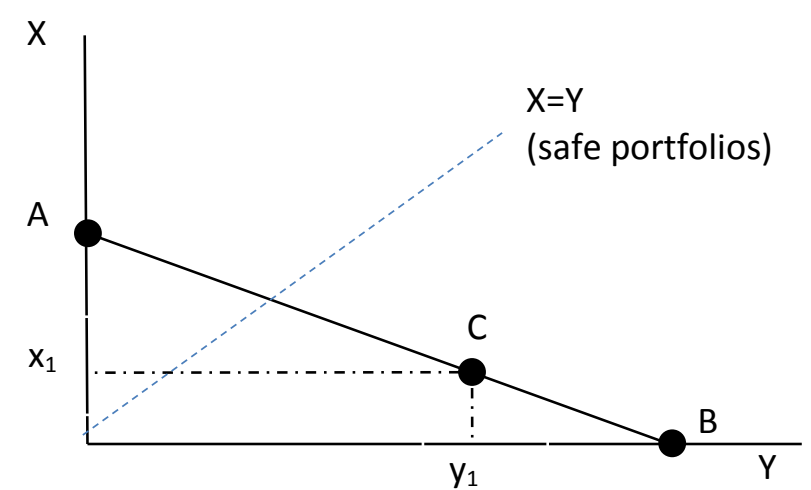

Figure 1

Sample Stimuli 


\section{Critical cost to efficiency}
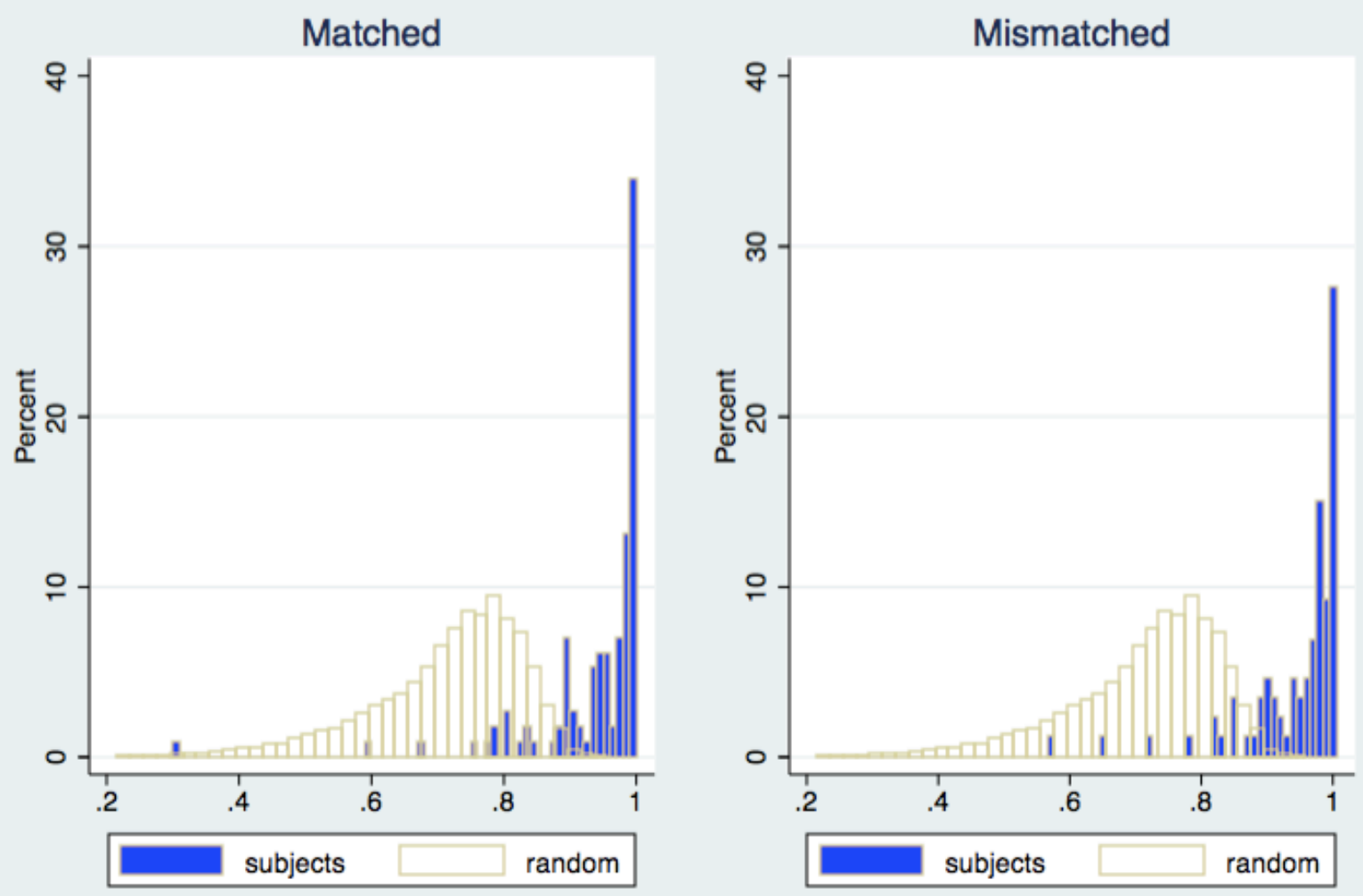

Figure 2

Distribution of Critical Cost to Efficiency Index (CCEI) for Matched and Mismatched subjects (blue bars) and for random choices (yellow bars) 


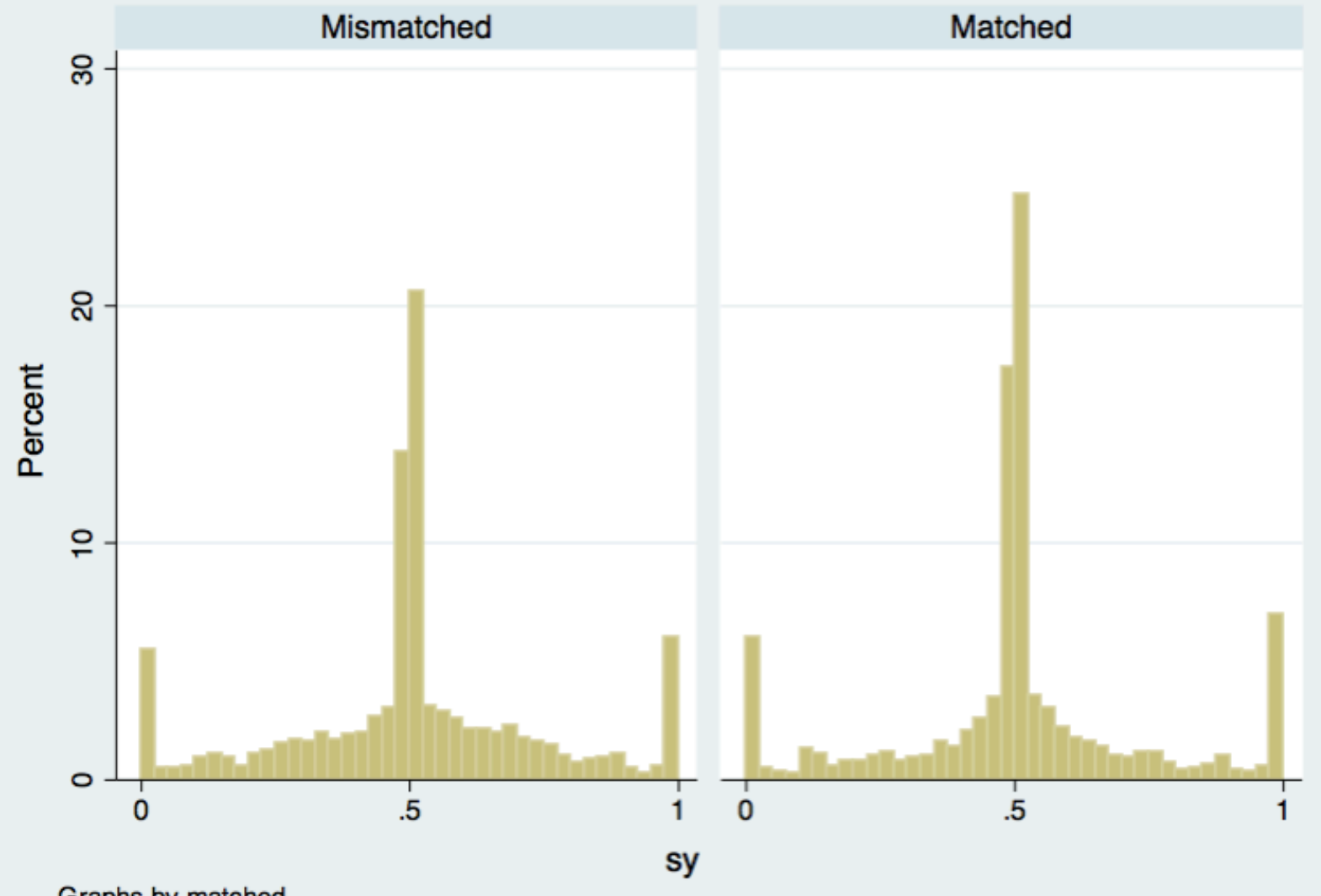

Graphs by matched

Figure 3

Share of Assets in Y for all relative prices-Matched and Mismatched subjects 


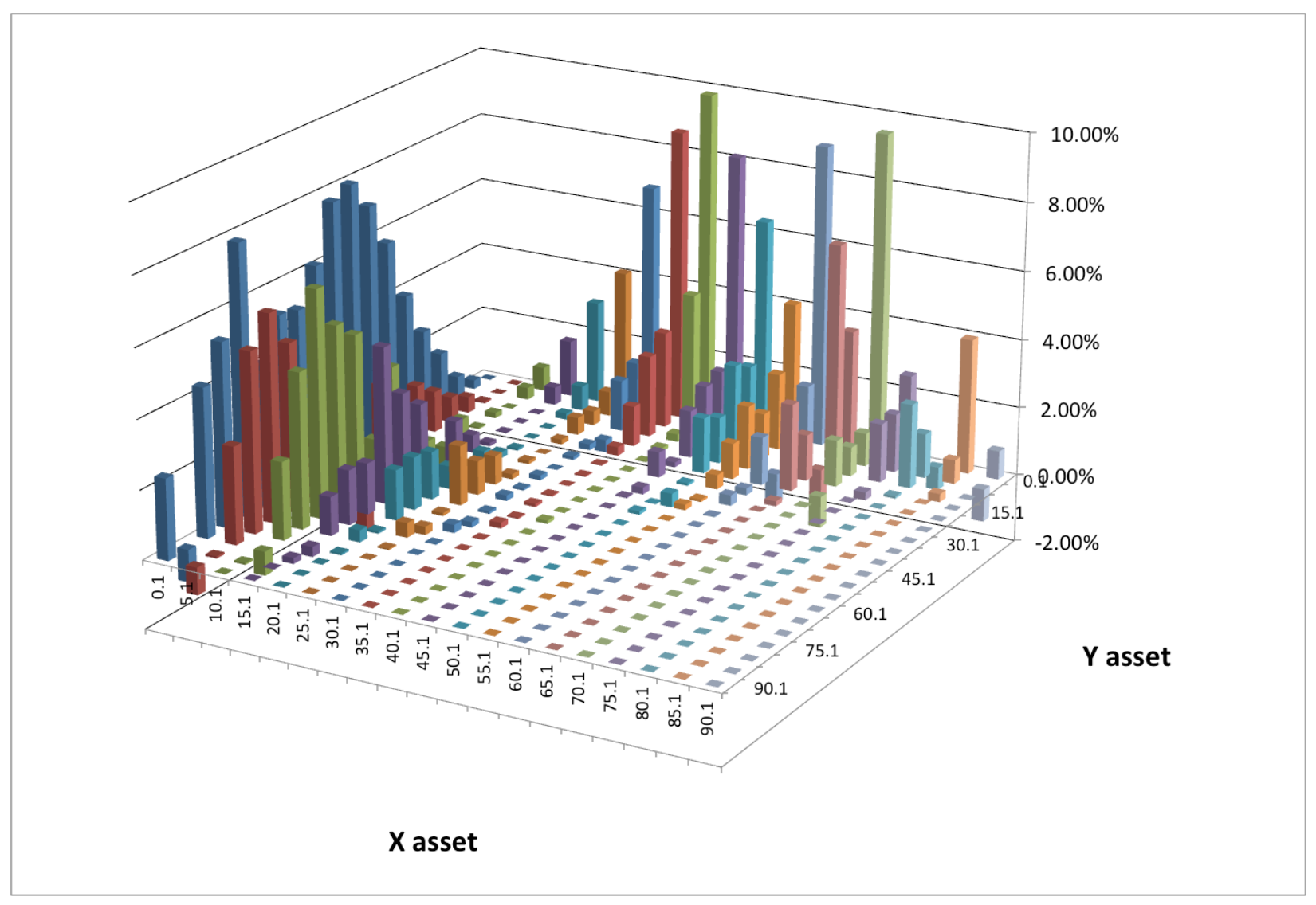

Figure 4: Differences in certainty equivalents (CE) for different asset bundles as a proportion of the expected value of the lottery for mismatched versus matched subjects

Table 1

Sample Size Per Design Cell

Morning Session

Evening Session

\begin{tabular}{|c|c|c|}
\cline { 2 - 3 } Morning-type & $32+22=54$ & $29+23=52$ \\
\cline { 2 - 3 } Evening-type & $24+11=35$ & $33+28=61$ \\
\cline { 2 - 3 } & $\begin{array}{l}\text { Total sample size }=202 \\
\text { Matched obs }=115, \text { Mismatched obs }=87\end{array}$ \\
\cline { 2 - 2 }
\end{tabular}

Note: (Site $1+$ Site $2=$ total sample size), Depleted Cognitive Resource cells shaded 
Table 2

Descriptive Statistics of Sample

\begin{tabular}{|l|c|c|c|}
\hline & $\begin{array}{c}\text { Circadian } \\
\text { Matched } \\
\text { (n=114) }\end{array}$ & $\begin{array}{c}\text { Circadian } \\
\text { Mismatched } \\
\text { (n=88) }\end{array}$ & $\begin{array}{c}\text { p-value for diff } \\
\text { in means test } \\
\text { (t-test) }\end{array}$ \\
\hline Pre-experiment survey & & & \\
\hline Avg hours of sleep & 6.8 & 6.8 & 0.9195 \\
\hline Hours slept last night & 6.8 & 6.8 & 0.8048 \\
\hline Optimal hours of sleep & 8.0 & 7.9 & 0.8357 \\
\hline $\begin{array}{l}\text { Epworth trait-level sleepiness score } \\
\text { (0-24, higher numbers indicate chronic fatigue) }\end{array}$ & 7.6 & 7.4 & 0.5855 \\
\hline & & & \\
\hline Post-experiment survey & & 6.7 & 0.0382 \\
\hline Avg hours of sleep over last 7 days & 7.1 & 6.2 & 0.0515 \\
\hline Hours slept last night & 6.7 & 8.0 & 0.8150 \\
\hline Optimal hours of sleep & 8.0 & 8.4 & 0.3600 \\
\hline $\begin{array}{l}\text { Epworth trait-level sleepiness score } \\
\text { (0-24, higher numbers indicate chronic fatigue) }\end{array}$ & 8.0 & & \\
\hline & & & \\
\hline Manipulation Checks & & 5.7 & 0.0000 \\
\hline $\begin{array}{l}\text { Avg state-level sleepiness rating during experiment } \\
\text { (Karolinska scale) }\end{array}$ & 4.5 & & \\
\hline (1=extremely alert, 9=extremely sleepy) & & 1.7 & 0.0904 \\
\hline $\begin{array}{l}\text { Subjective sleep deprivation } \\
\text { (optimal sleep-last night's sleep) }\end{array}$ & 1.3 & 7.3 & 0.8959 \\
\hline $\begin{array}{l}\text { Avg decision response time in seconds } \\
\text { (std dev of mean in parentheses) }\end{array}$ & 9.4 & & \\
\hline $\begin{array}{l}\text { Avg std dev of response time } \\
\text { (in seconds) }\end{array}$ & $(4.07)$ & & \\
\hline
\end{tabular}


Table 3

Consistency versus Violations of Payoff Dominance: Matched vs. Mismatched subject Fisher tests of proportions for each category (number listed, proportion of sample in parenthesis)

\begin{tabular}{|c|c|c|c|}
\hline Category & $\begin{array}{l}\text { All } \ln \left(\frac{P_{x}}{P_{Y}}\right) \\
(n=10,100)\end{array}$ & $\begin{array}{c}\left|\ln \left(\frac{P_{x}}{P_{Y}}\right)\right|<.10 \\
(n=2,203)\end{array}$ & $\begin{array}{c}\left|\ln \left(\frac{P_{x}}{P_{Y}}\right)\right|<.05 \\
(n=1,156)\end{array}$ \\
\hline \multirow{2}{*}{$\begin{array}{c}\text { Relatively cheap X } \\
\text { Dominance consistent choice }\end{array}$} & $\begin{array}{l}\mathrm{N}_{\text {Matched }}=2128 \\
(73.46 \%)\end{array}$ & $\begin{array}{c}N_{\text {Matched }}=371 \\
(57.61 \%)\end{array}$ & $\begin{array}{c}\mathrm{N}_{\text {Matched }}=193 \\
(53.76 \%)\end{array}$ \\
\hline & $\begin{array}{c}\mathrm{N}_{\text {Mismatched }}=1647 \\
(74.42 \%)\end{array}$ & $\begin{array}{c}N_{\text {Mismatched }}=295 \\
(59.84 \%)\end{array}$ & $\begin{array}{c}\mathrm{N}_{\text {Mismatched }}=143 \\
(55.21 \%)\end{array}$ \\
\hline \multirow{2}{*}{$\begin{array}{c}\text { Relatively cheap } Y \\
\text { Dominance consistent choice }\end{array}$} & $\begin{array}{c}\mathrm{N}_{\text {Matched }}=2208 \\
(77.39 \%)\end{array}$ & $\begin{array}{c}N_{\text {Matched }}=393 \\
(63.80 \%)\end{array}$ & $\begin{array}{c}\mathrm{N}_{\text {Matched }}=204 \\
(63.75 \%)\end{array}$ \\
\hline & $\begin{array}{c}\mathrm{N}_{\text {Mismatched }}=1687 \\
(78.94 \%)\end{array}$ & $\begin{array}{c}\mathrm{N}_{\text {Mismatched }}=311 \\
(69.11 \%)\end{array}$ & $\begin{array}{c}\mathrm{N}_{\text {Mismatched }}=140 \\
(64.22 \%)\end{array}$ \\
\hline \multirow{2}{*}{$\begin{array}{c}\text { Relatively cheap } \mathrm{X} \\
\text { Dominance violated choice }\end{array}$} & $\begin{array}{c}\mathrm{N}_{\text {Matched }}=769 \\
(26.54 \%)\end{array}$ & $\begin{array}{c}\mathrm{N}_{\text {Matched }}=273 \\
(42.39 \%)\end{array}$ & $\begin{array}{c}\mathrm{N}_{\text {Matched }}=166 \\
(46.24 \%)\end{array}$ \\
\hline & $\begin{array}{c}\mathrm{N}_{\text {Mismatched }}=566 \\
(25.58 \%)\end{array}$ & $\begin{array}{c}\mathrm{N}_{\text {Mismatched }}=198 \\
(40.16 \%)\end{array}$ & $\begin{array}{c}\mathrm{N}_{\text {Mismatched }}=116 \\
(44.79 \%)\end{array}$ \\
\hline \multirow{2}{*}{$\begin{array}{c}\text { Relatively cheap } Y \\
\text { Dominance violated choice }\end{array}$} & $\begin{array}{c}\mathrm{N}_{\text {Matched }}=645 \\
(22.61 \%)\end{array}$ & $\begin{array}{c}\mathrm{N}_{\text {Matched }}=223 \\
(36.20 \%)\end{array}$ & $\begin{array}{c}\mathrm{N}_{\text {Matched }}=116 \\
(36.25 \%)\end{array}$ \\
\hline & $\begin{array}{c}N_{\text {Mismatched }}=450 \\
(21.06 \%)\end{array}$ & $\begin{array}{c}\mathrm{N}_{\text {Mismatched }}=139 \\
(30.89 \%)\end{array}$ & $\begin{array}{c}\mathrm{N}_{\text {Mismatched }}=78 \\
(35.78 \%)\end{array}$ \\
\hline FISHER'S EXACT TEST & 0.465 & 0.247 & 0.948 \\
\hline
\end{tabular}


Table 4

Interquantile regression on share in $\mathrm{Y}(\mathrm{Y} /(\mathrm{Y}+\mathrm{X}))$

\begin{tabular}{lcc}
\hline VARIABLES & $\begin{array}{c}\text { No } \\
\text { bootstrap }\end{array}$ & $\begin{array}{c}\text { Bootstrap } \\
\text { at the } \\
\text { subject level }\end{array}$ \\
\hline Mismatched & $0.085^{* * *}$ & $0.085^{* *}$ \\
& {$[0.012]$} & {$[0.042]$} \\
Constant & $(0.000)$ & $(0.041)$ \\
& $0.107^{* * *}$ & $0.107^{* * *}$ \\
& {$[0.008]$} & {$[0.023]$} \\
& $(0.000)$ & $(0.000)$ \\
Observations & 10,094 & 10,094 \\
\hline
\end{tabular}

Standard errors in brackets, $\mathrm{p}$-values in parentheses. Only decisions over positive prices are included. $* * * p<0.01,{ }^{* *} p<0.05, * p<0.10$

Table 5

Tobit estimates of the certainty equivalents as a proportion of the expected value of the lottery

\begin{tabular}{lccccc}
\hline \multirow{2}{*}{ VARIABLES } & $\begin{array}{c}\text { All } \\
\text { lotteries }\end{array}$ & $\mathrm{X}<\mathrm{Y}$ & $\begin{array}{c}|\ln (\mathrm{x} / \mathrm{y})|> \\
|\ln (1 / 1.1)|\end{array}$ & $\begin{array}{c}|\ln (\mathrm{x} / \mathrm{y})|> \\
|\ln (1 / 10)|\end{array}$ & $\begin{array}{c}|\ln (\mathrm{x} / \mathrm{y})|> \\
|\ln (1 / 100)|\end{array}$ \\
\hline \multirow{3}{*}{ Mismatched } & & & & & \\
& $0.044^{* * *}$ & $0.044^{* * *}$ & 0.044 & 0.067 & 0.088 \\
& {$[0.006]$} & {$[0.006]$} & {$[0.033]$} & {$[0.049]$} & {$[0.061]$} \\
Constant & $(0.000)$ & $(0.000)$ & $(0.178)$ & $(0.172)$ & $(0.148)$ \\
& $3.188^{* * *}$ & $3.186^{* * *}$ & $1.890^{* * *}$ & $2.174 * * *$ & $2.188^{* * *}$ \\
& {$[0.007]$} & {$[0.007]$} & {$[0.129]$} & {$[0.167]$} & {$[0.149]$} \\
& $(0.000)$ & $(0.000)$ & $(0.000)$ & $(0.000)$ & $(0.000)$ \\
& & & & & \\
Observations & 72,922 & 71,710 & 65,852 & 10,504 & 6,868 \\
\hline
\end{tabular}

Dummy variables per lottery included, 202 clusters (subjects)

Robust standard errors in brackets, $p$-values in parentheses 
Table 6

Price elasticity on $\mathrm{y} /(\mathrm{y}+\mathrm{x})$

VARIABLES

$\ln \left(p_{y} / p_{x}\right)$

$-0.160 * * *$

[0.007]

(0.000)

$\ln \left(\mathrm{p}_{\mathrm{y}} / \mathrm{p}_{\mathrm{x}}\right) *$ Mismatched

$-0.026 * *$

[0.012]

(0.028)

Mismatched

0.002

[0.007]

(0.710)

Constant

$0.503 * * *$

[0.005]

(0.000)

Observations

10,094

R-squared

0.387

Robust standard errors in brackets, $p$-value in parentheses. Errors clustered at the subject level. Only decisions over positive prices are included. ${ }^{* *} p<0.01,{ }^{* *} p<0.05,{ }^{*} p<0.10$. 


\section{APPENDIX - SUBJECT INSTRUCTIONS}

\section{INSTRUCTIONS}

This is an experiment in decision-making. Your payoffs will depend partly on your decisions and partly on chance. Your payoffs will not depend on the decisions of the other participants in the experiment. Please pay careful attention to the instructions as a considerable amount of money is at stake.

The entire experiment should be complete within an hour and a half. At the end of the experiment you will be paid privately. Your total payoff in this experiment will consist of \$5 as a participation fee (simply for showing up on time), plus whatever payoff you receive from the decision experiment. Details of how your payoff will depend on your decisions will be provided below.

During the experiment we will speak in terms of experimental tokens instead of dollars. Your payoffs will be calculated in terms of tokens and then translated at the end of the expeirment into dollars at the following rate:

$$
2 \text { Tokens }=1 \text { Dollar }
$$

\section{The decision problem}

In this experiment, you will participate in 50 independent decision problems that share a common form. This section describes in detail the process that will be repeated in all decision problems and the computer program that you will use to make your decisions.

In each decision problem you will be asked to allocate tokens between two accounts, labeled $x$ and $y$. The $x$ account corresponds to the $x$-axis and the $y$ account corresponds to the $y$ axis in a two-dimensional graph. Each choice will involve choosing a point on a line representing possible token allocations. Examples of lines that you might face appear in the graph below. Many lines are shown on the same graph to highlight that there will be a variety of different lines you could face, but each decision you make will involve only one line on the graph, as you will see further in these instructions. 


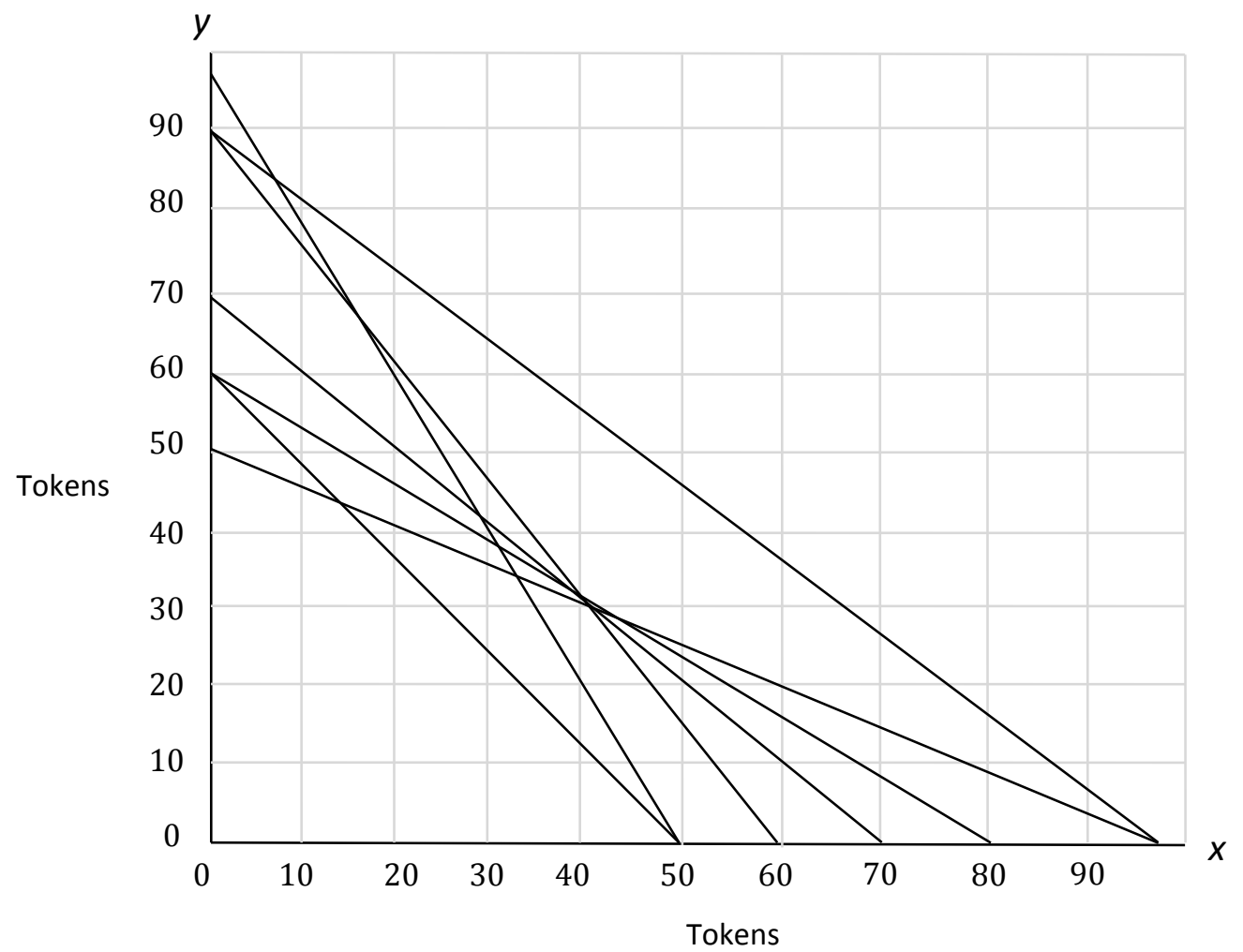

In each choice, you may choose any $x$ and $y$ pair that is on the line. For example, as illustrated in the next graph below, choice $\boldsymbol{A}$ represents a decision to allocate 14 tokens in the $x$ account and 70 tokens in the $y$ account. Another possible allocation is $\boldsymbol{B}$, in which you allocate 40 tokens in the $x$ account and 30 tokens in the $y$ account. 


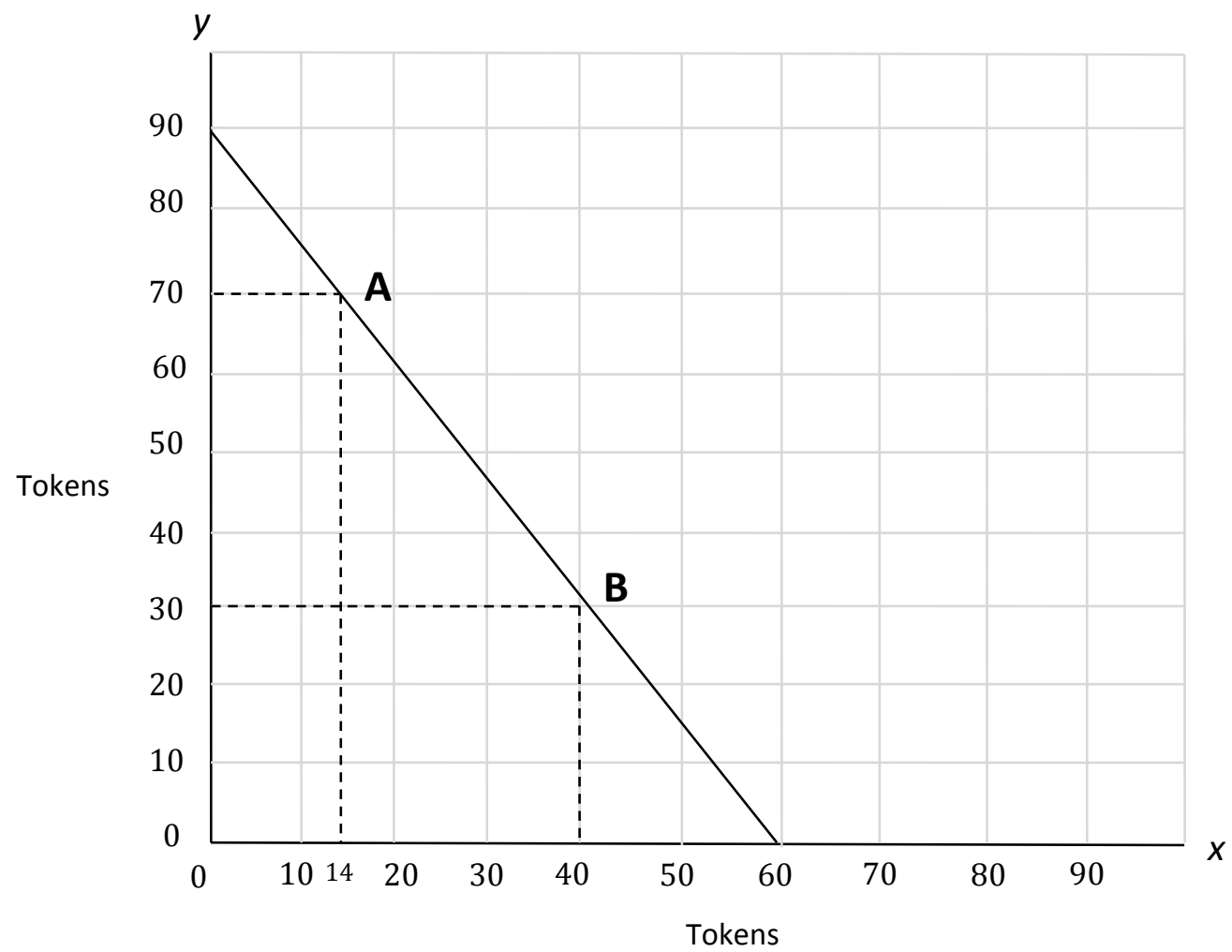

Each decision problem will start by having the computer select such a line randomly from the set of lines that intersect with at least one of the axes at 50 or more tokens but with no intercept exceeding 100 tokens. The lines selected for you in different decision problems are independent of each other and independent of the lines selected for any of the other participatns in their decision problems.

To choose an allocation in each decision problem, use the mouse to move the pointer on the computer screen to the allocation that you desire. When you are ready to make your decision, left-click to enter your chosen allocation. After that, confirm your decision by clicking on the Submit button that will appear after your decision is made. Note that you can choose only $x$ and $y$ combinations that are on the line (you may also choose either endpoint on any line if you so desire). The next graph shows a picture of the actual decision screen you will see in the experiment. Notice that where you position the pointer on the line will highlight exactly what combination of $x$ and $y$ are at that location on the line. This same information is also shown in the information area to the right of the graph (the example graph shows additional information that will be discussed next in these instructions. It also indicates a 20 round experiment, although today's experiment will be 50 rounds in length). 


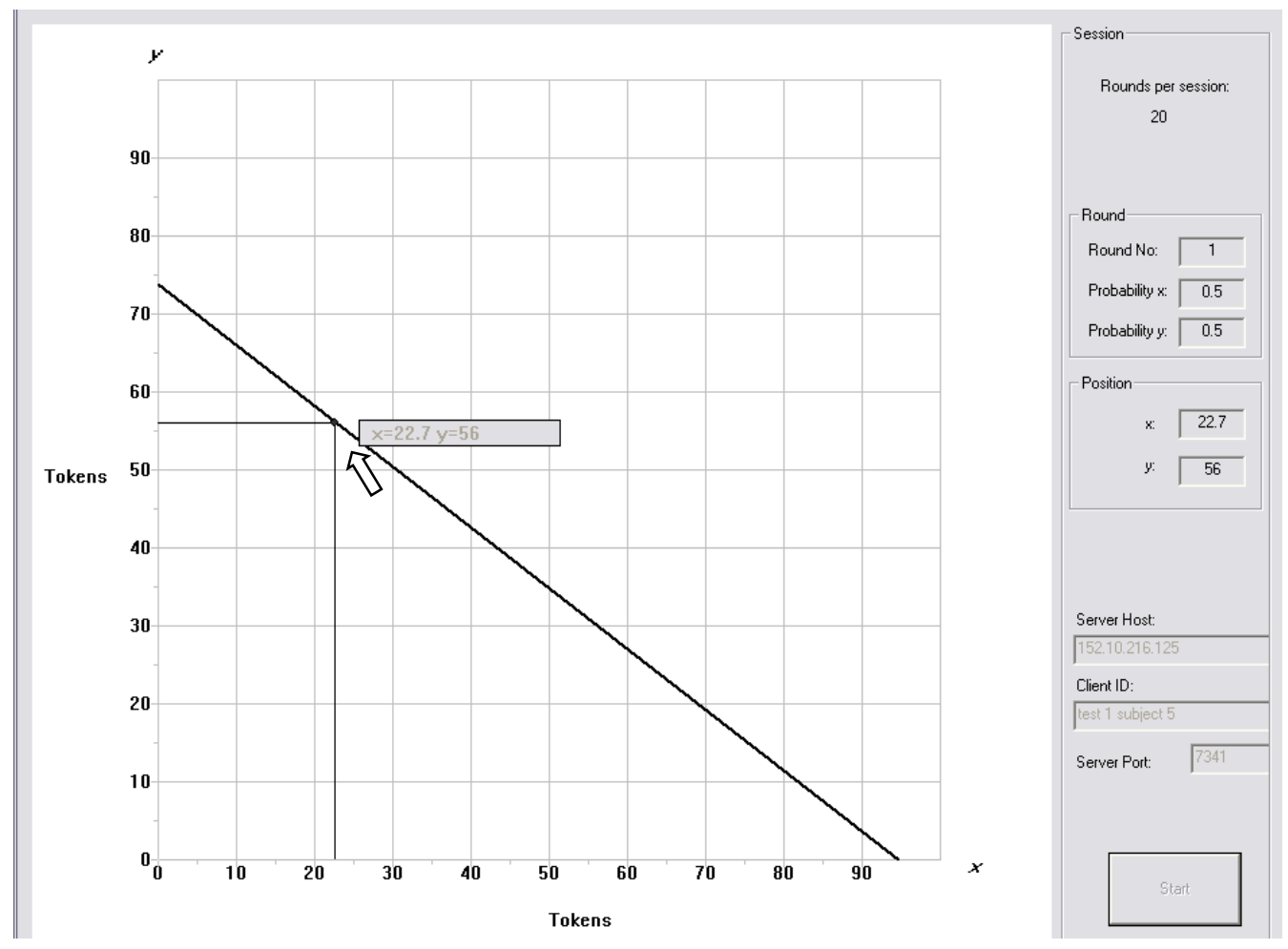

Once you have confirmed your choice for that decision round, press the OK button. Your payoff in each decision round is determined by the number of tokens in your $x$ account and the number of tokens in your $y$ account. At the end of the round, the computer will randomly select one of the accounts, $x$ or $y$. There is an equal chance that either account will be selected, and this random selection occurs separately and independently for each participant. You will only receive as payment the number of tokens you allocated to the account that was chosen. (In the example graph directly above, if account $x$ is selected you would receive 22.7 tokens, and if account $y$ is selected you would receive 56 tokens). The random selection of account $x$ or $y$ in a decision round will not be shown to you until the very end of the experiment.

Once a decision round is finished, you will be asked to make an allocation in another independent decision. This process will be repeated until all 50 decision rounds are completed. At the end of the last round, you will be informed that the experiment has ended.

\section{Your earnings}

Your earnings in the experiment are determined as follows. At the end of the experiment, the computer will randomly select one decision round to carry out (that is, 1 out of 50). The round selected depends solely upon chance, and it is equally likely that any round will be chosen. Once a round is chosen, you will receive the number of tokens you allocated to the account ( $x$ or $y$ ) that was randomly selected for that round. Keep in mind that there is an equal chance that account $x$ or $y$ will be chosen for your token payoff in any given round.

The round selected, your choice and your payment (in terms of tokens) will be shown in the large window that appears at the center of the program dialog window. At the end of the 
experiment, the tokens will be converted into money. Each token will be worth 0.5 dollars (in other words, your "tokens" payoff will be divided by 2 to get your payoff in dollars). Your final cash earnings in the experiment will be your earnings in the round selected plus the \$5 show-up fee. You will receive your payment as you leave the experiment.

\section{$\underline{\text { Rules }}$}

Please do not share your decisions with anyone else in today's experiment, please do not talk with anyone during the experiment, and please remain silent until everyone is finished. If there are no further questions, you are ready to start, and an experimenter will start your experiment program. 